\title{
Efeito do ultrassom pulsado e do ultrassom contínuo associado a exercícios em pacientes com osteoartrite de joelho: estudo piloto
}

\author{
Effect of pulsed ultrasound and continuous ultrasound linked to exercise in patients \\ with knee osteoarthritis: pilot study
}

Karina Paz Carlos', Bruno dos Santos Belli', Patrícia Pereira Alfredo²

RESUMO I A osteoartrite (OA) é uma doença crônica associada à morbidade significante. O objetivo deste estudo foi comparar o exercício isolado ao ultrassom pulsado (USP) e contínuo (USC) associados a exercício na redução da dor, melhora da amplitude de movimento (ADM), força muscular (FM), qualidade de vida (QV) e funcionalidade de pacientes com OA de joelhos. Trinta indivíduos, 50 a 75 anos, OA grau 2-4, foram randomizados: Grupo USC (USC + exercícios), Grupo USP (USP + exercícios) e Grupo EXE (exercícios). Os grupos foram avaliados antes e após o tratamento. A intervenção foi realizada três vezes por semana durante oito semanas: nas quatro primeiras foi aplicado USC ou USP e, nas demais foram realizados os exercícios. O Grupo EXE realizou exercícios durante oito semanas. Para análises intragrupos, utilizou-se o teste de Wilcoxon e, intergrupos, o teste de Kruskall-Walis. Na comparação intragrupos, o Grupo USC apresentou melhora significativa $(p \leq 0,05)$ nas variáveis da Western Ontario and McMaster Universities Osteoarthritis (WOMAC) dor, função e escore total; o Grupo USP na dor repouso, ADM, FM e nas variáveis da WOMAC dor, função e escore total; já, o Grupo EXE na mobilidade e ADM. Na comparação intergrupos, o Grupo USC apresentou melhora significativa ( $p \leq 0,05)$ quando comparado aos demais grupos na dor repouso, ADM, escores função e total da WOMAC e o Grupo EXE na dor da WOMAC. A associação do USC a exercícios foi mais efetiva na melhora da dor, ADM, função e QV em pacientes com OA de joelho.

Descritores | osteoartrite do joelho; terapia por exercício; terapia por ultrassom.

\begin{abstract}
I Osteoarthritis (OA) is a chronic disease associated with significant morbidity. The aim of this study was to compare the isolated exercises with association of pulsed (PUS) and continuous (CUS) ultrasound with exercise in reducing pain, improving range of motion (ROM), muscle strength (MS), quality of life (QL) and functionality of patients with knee OA. Thirty individuals, 50 to 75 years old, 2-4 OA grade, were randomized: Group CUS (CUS + exercise), Group PUS (PUS + exercise) and EXE group (exercises). The groups were evaluated before and after treatment. The intervention was performed three times a week for eight weeks: in the first four weeks, it was applied CUS or PUS, and in other weeks, exercises were carried out. The EXE group performed exercises for eight weeks. For intra-group analysis, the Wilcoxon test was used and intergroup analysis, the Kruskall-Walis test was used. Within the groups, the USC group showed significant improvement ( $p \leq 0.05)$ in variables of Western Ontario and McMaster Universities Osteoarthritis (WOMAC) pain, function and total score; the Group USP in pain-rest, ROM, MS, variables of WOMAC pain, function and total score; and EXE Group in mobility and ROM. In intergroup comparison, the USC group showed significant improvement ( $p \leq 0.05)$ when compared to other groups in pain-rest, ROM, variables of WOMAC function and total score and EXE group in WOMAC-pain. The association of USC and exercise was more effective in reducing pain, ROM, function and $Q L$ in patients with knee $O A$.
\end{abstract}

Keywords | osteoarthritis, knee; exercise therapy; ultrasonic therapy.

Estudo desenvolvido no Ambulatório do Município de Barueri - Barueri (SP), Brasil.

'Aluno do Curso de Fisioterapia da Pontifícia Universidade Católica de São Paulo (PUC-SP) - São Paulo (SP), Brasil.

${ }^{2}$ Professora Doutora e Coordenadora do Curso de Fisioterapia da PUC-SP - São Paulo (SP), Brasil. 


\section{INTRODUÇÃO}

A osteoartrite (OA) é uma doença musculoesquelética, sendo uma das causas mais comuns de limitação funcional e dependência ${ }^{1}$. Afeta cerca de $50 \%$ das pessoas com idade acima de 65 anos $^{2}$. No Brasil, é a segunda doença que mais resulta em auxílio doença $(10,5 \%)$ e a quarta a determinar aposentadoria $(6,2 \%)^{3}$. Destacamse as OA de quadril e joelho por serem particularmente mais incapacitantes.

A dor é o principal sintoma da OA, piorando com o movimento articular e ao final do dia ${ }^{5}$. A diminuição da força está presente nos grupos musculares que são responsáveis pela estabilização das articulações com $\mathrm{OA}$, como o quadríceps ${ }^{6}$, causando progressiva perda de função. Atuais estratégias terapêuticas são essencialmente destinadas ao controle da dor e melhora da função articular, minimizando a incapacidade ${ }^{7}$.

Como parte da abordagem fisioterapêutica, estão exercícios, cujos benefícios são comprovados na restauração da amplitude de movimento (ADM), força muscular (FM), melhora da dor e da realização das atividades de vida diária ${ }^{8,9}$.

O ultrassom terapêutico (US) é uma das modalidades físicas sugeridas pelo Colégio Americano de Reumatologia ${ }^{10}$ e Liga Europeia contra o Reumatismo ${ }^{9}$ para o tratamento da dor e perda funcional causada pela OA. $\mathrm{Na}$ literatura observam-se resultados conflitantes em relação aos benefícios do tratamento com US pulsado ou contínuo em pacientes com OA de joelho e são escassos estudos que especifiquem quais exercícios são mais seguros ou quais intensidades e frequências devem ser utilizadas. Sendo assim, este estudo teve como objetivo comparar o efeito do US pulsado ao do US contínuo, associado a exercícios, ao exercício isolado, na redução da dor, na melhora da mobilidade articular, FM, qualidade de vida (QV) e funcionalidade de pacientes com OA de joelhos.

\section{METODOLOGIA}

\section{Amostra}

Participaram do estudo 30 pacientes com OA de joelho, ambos os sexos, idade entre 50 e 75 anos.

Foram critérios de inclusão: graus $2-4$ de $\mathrm{OA}^{11}$, dor no joelho e redução funcional nos últimos três meses. Foram critérios de exclusão: diabetes mellitus, demência, câncer, hipertensão arterial sistêmica não controlada, obesidade mórbida, $\mathrm{OA}$ de quadril sintomática e que faziam uso de anti-inflamatórios ou ansiolíticos nos últimos seis meses antes da avaliação inicial.

Os participantes foram orientados a não utilizar nenhum tipo de medicamento analgésico ou anti-inflamatório durante o estudo.

\section{Randomização}

Os sujeitos foram randomizados aleatoriamente em três grupos: Ultrassom Contínuo (USC), Ultrassom Pulsado (USP) e Exercícios (EXE) por um pesquisador que não estava envolvido no tratamento e na avaliação. $\mathrm{O}$ paciente e o fisioterapeuta avaliador não tiveram acesso ao resultado.

\section{Procedimentos}

\section{Avaliação (Fisioterapeuta 1)}

Foram avaliados:

a) Dados pessoais: anamnese;

b) QV: pelo Questionário Western Ontario and McMaster Universities Osteoarthritis (WOMAC)12. Um instrumento de autoavaliação que avalia dor, rigidez articular e atividade física. Quanto maior o escore, maior é o impacto da OA na qualidade de vida;

c) Funcionalidade: pelo Questionário Lequesne ${ }^{13}$. Um índice composto de 11 questões sobre dor, desconforto e função. As pontuações variam de 0 a 2 e, quanto maior o escore, maior o acometimento;

d) Dor: pela Escala Visual Analógica (EVA) ${ }^{14}$. Consiste em uma reta de $10 \mathrm{~cm}$ de comprimento desprovida de números, na qual há apenas a indicação no extremo esquerdo de 'ausência de dor' e no extremo direito de 'dor insuportável'. Quanto maior o escore, maior a intensidade de dor;

e) Amplitude de movimento de flexão do joelho: pelo goniômetro universal com o paciente em pronação ${ }^{15}$;

f) FM do quadríceps: pelo dinamômetro portátil modelo Lafayette ${ }^{\circledR}$, com o indivíduo sentado com 10, 60 e $90^{\circ}$ de flexão do joelho (angulação controlada com goniômetro). Esse dinamômetro manual tem excelente reprodutibilidade para avaliação da FM do quadríceps ${ }^{16}$

g) Mobilidade: pelo Timed Get Up and Go (TGUG) ${ }^{17}$ que quantifica em segundos o tempo que o indivíduo necessita para realizar a tarefa de levantar de uma 
cadeira, caminhar três metros, virar, voltar rumo à cadeira e sentar novamente.

\section{Intervenção (Fisioterapeuta 2)}

Foi utilizado o modelo de US Sonopulse Compact-1 MHz da Ibramed, área de radiação efetiva (ERA) de $3,5 \mathrm{~cm}^{2}$, potência média de saída de $7 \mathrm{~W}$, tempo de aplicação de 5 min no lado medial e 5 min no lado lateral do joelho.

Foi aplicado em três sessões semanais por quatro semanas. Nas quatro semanas seguintes, os pacientes receberam apenas sessões de exercícios três vezes por semana.

\section{Grupo Ultrassom Contínuo associado a Exercícios}

Foi utilizado US com intensidade de $1,5 \mathrm{~W} / \mathrm{cm}^{2}$ (média espacial), modo contínuo (100\%).

\section{Grupo Ultrassom Pulsado associado a Exercicios}

Foi utilizado US com intensidade de $2,5 \mathrm{~W} / \mathrm{cm}^{2}$ (média temporal e espacial), modo pulsado (20\%), frequência de repetição de pulso a $100 \mathrm{~Hz}$.

\section{Grupo Exercícios}

Os pacientes receberam sessões de exercícios, três vezes por semana durante oito semanas.

\section{Protocolo de exercícios}

Todos os grupos realizaram o mesmo programa de exercícios isotônicos, sendo respeitada a limitação de cada paciente. Cada exercício foi realizado em duas séries com 30 repetições. Cada sessão de exercícios teve duração de 45 minutos: 10 minutos de aquecimento (esteira ou bicicleta ergométrica), 30 minutos de exercícios, 5 minutos de alongamento (isquiotibiais, quadríceps, adutores do quadril e gastrocnêmios).

\section{Análise estatística}

Antes de se realizarem as análises, os valores foram padronizados:

- Variáveis onde a diminuição indica melhora: (AV1-AV2)/AV1;

- Variáveis onde o aumento indica melhora: (AV2-AV1)/AV1.

Para a comparação das características demográficas e clínicas dos pacientes dos Grupos Ultrassom Contínuo
Associado a Exercícios, Ultrassom Pulsado Associado a Exercícios e Exercícios (USC, USP e EXE)no momento da linha de base foi realizado a analise de variância (Anova) one way.

Para as análises intragrupos foi utilizado o teste não paramétrico de Wilcoxon e intergrupos o teste não paramétrico de Kruskall-Walis. O teste de múltipla comparação de Dunn foi aplicado para identificar as diferenças entre os grupos.

As análises foram conduzidas utilizando-se $\mathrm{O}$ Statistical Package for Social Sciences (SPSS, versão 17) e admitido um nivel de significancia de 5\%.

\section{RESULTADOS}

A Tabela 1 mostra a distribuição dos três grupos no início do tratamento. Não houve diferença estatisticamente significante $(p>0,05)$ entre os grupos para as variáveis: idade, peso, altura, índice de massa corporal (IMC), sexo e grau de OA.

\section{Comparação intragrupos}

Na Tabela 2 são apresentados os intervalos de confiança (IC) do grupo USC. Houve diferença estatisticamente significante, indicando melhora, nas variáveis WOMAC dor ( $p=0,014)$, WOMAC função $(p=0,008)$ e WOMAC total $(\mathrm{p}=0,014)$.

$\mathrm{Na}$ Tabela 3 são apresentados os IC do grupo USP. Houve diferença estatisticamente significante, indicando melhora, nas variáveis dor ao repouso $(\mathrm{p}=0,008)$, WOMAC dor $(p=0,009)$, WOMAC função $(p=0,006)$ e WOMAC total $(\mathrm{p}=0,008)$.

$\mathrm{Na}$ Tabela 4 são apresentados os IC do grupo EXE. Houve diferença estatisticamente significante, indicando melhora, nas variáveis mobilidade $(\mathrm{p}=0,019), \mathrm{ADM}$ $(\mathrm{p}=0,025)$.

\section{Comparação intergrupos}

A Tabela 5 mostra a comparação das medianas das variáveis dor, mobilidade, ADM, FM, variáveis da WOMAC e funcionalidade entre os Grupos USC, USP e EXE, nas avaliações 1 e 2 . Houve diferença estatisticamente significante entre os grupos nas variáveis dor ao repouso $(p=0,03)$, WOMAC dor $(p=0,01)$, WOMAC função $(p=0,03)$ e WOMAC total $(p=0,01)$. O Grupo EXE e o USC foram mais eficazes na melhora das variáveis dor 
Tabela 1. Características demográficas e clínicas dos pacientes dos Grupos Ultrassom Contínuo, Ultrassom Pulsado e Exercício, momento da linha de base

\begin{tabular}{|c|c|c|c|c|}
\hline \multicolumn{5}{|c|}{ Grupos } \\
\hline \multirow[t]{2}{*}{ Variáveis } & USC $(n=10)$ & USP $(n=10)$ & $\operatorname{EXE}(n=10)$ & \multirow[t]{2}{*}{ Valor $\mathrm{p}^{*}$} \\
\hline & Média (DP) & Média (DP) & Média (DP) & \\
\hline Idade (anos) & $63,9(6,31)$ & $63,4(4,59)$ & $62,7(8,71)$ & 0,924 \\
\hline Peso (kg) & $82,11(16,94)$ & $73,69(7,53)$ & $79,8(11,06)$ & 0,312 \\
\hline Altura (m) & $1,59(0,11)$ & $1,63(0,07)$ & $1,6(0,08)$ & 0,799 \\
\hline \multirow[t]{2}{*}{$\mathrm{IMC}\left(\mathrm{kg} / \mathrm{m}^{2}\right)$} & $31,81(4,06)$ & $27,78(3,83)$ & $31,09(3,25)$ & 0,052 \\
\hline & n (\%) & n (\%) & $\mathrm{n}(\%)$ & \\
\hline \multicolumn{5}{|l|}{ Sexo } \\
\hline Feminino & $7(70)$ & $7(70)$ & $7(70)$ & \\
\hline Masculino & $3(30)$ & $3(30)$ & $3(30)$ & \\
\hline \multicolumn{5}{|l|}{ Grau OA } \\
\hline Joelho sem OA & $1(5)$ & $3(15)$ & $\mathrm{O}(\mathrm{O})$ & \\
\hline 2 & $5(25)$ & $3(15)$ & $11(55)$ & \\
\hline 3 & $9(45)$ & $10(50)$ & $7(35)$ & \\
\hline 4 & $5(25)$ & $4(20)$ & $2(10)$ & \\
\hline
\end{tabular}

DP: desvio padrão; USC: Grupo Ultrassom Contínuo; USP: Grupo Ultrassom Pulsado; EXE: Grupo Exercício; IMC: índice de massa corporal; OA: osteoartrite; *Teste ANOVA - one way

Tabela 2. Escores padronizados da dor, mobilidade, amplitude de movimento, força muscular, variáveis da Western Ontario and McMaster Universities Osteoarthritis e funcionalidade nos dois tempos de avaliação do Grupo Ultrassom Contínuo

\begin{tabular}{|c|c|c|c|c|c|}
\hline Variáveis & Mediana & Est. Wilcoxon & Lim. Inf. (IC95\%) & Lim. Sup. (IC95\%) & Valor $p$ \\
\hline \multicolumn{6}{|l|}{ Dor } \\
\hline AVD & 0,097 & 31 & $-0,098$ & 0,338 & 0,343 \\
\hline Repouso & 0,319 & 43 & $-0,094$ & 0,584 & 0,126 \\
\hline Mobilidade & 0,136 & 41 & $-0,077$ & 0,237 & 0,185 \\
\hline \multicolumn{6}{|l|}{ ADM } \\
\hline Flexão & 0,060 & 40 & $-0,015$ & 0,194 & 0,221 \\
\hline \multicolumn{6}{|l|}{ Força Muscular } \\
\hline $60^{\circ}$ & $-0,045$ & 21 & $-0,359$ & 0,345 & 0,541 \\
\hline $90^{\circ}$ & 0,207 & 38 & $-0,101$ & 0,511 & 0,308 \\
\hline $10^{\circ}$ & $-0,139$ & 19 & $-0,553$ & 0,262 & 0,415 \\
\hline \multicolumn{6}{|l|}{ WOMAC } \\
\hline Dor & 0,383 & 36 & 0,188 & 0,565 & $0,014^{*}$ \\
\hline Rigidez & 0,125 & 17 & $-0,143$ & 0,257 & 0,673 \\
\hline Função & 0,383 & 54 & 0,161 & 0,482 & $0,008^{*}$ \\
\hline Total & 0,337 & 52 & 0,115 & 0,492 & $0,014^{*}$ \\
\hline Funcionalidade & 0,036 & 27 & $-0,137$ & 0,188 & 0,636 \\
\hline
\end{tabular}

Est. Wilcoxon: valor crítico do teste de Wilcoxon; Lim. Inf. (IC): limite inferior do intervalo de confiança; Lim. Sup. (IC): limite superior do intervalo de confiança; AVD: atividades de vida diária; ADM: amplitude de movimento; WOMAC: Western Ontario and McMaster Universities Osteoarthritis; *teste de Wilcoxon

Tabela 3. Escores padronizados da dor, mobilidade, amplitude de movimento, força muscular, variáveis da Western Ontario and McMaster Universities Osteoarthritis e funcionalidade nos dois tempos de avaliação do Grupo Ultrassom Pulsado

\begin{tabular}{|c|c|c|c|c|c|}
\hline Variáveis & Mediana & Est. Wilcoxon & Lim. Inf. (IC95\%) & Lim. Sup. (IC95\%) & Valor $p$ \\
\hline \multicolumn{6}{|l|}{ Dor } \\
\hline AVD & 0,256 & 45 & $-0,014$ & 0,498 & 0,083 \\
\hline Repouso & 0,570 & 54 & 0,348 & 0,780 & $0,008^{*}$ \\
\hline Mobilidade & 0,057 & 34 & $-0,104$ & 0,233 & 0,541 \\
\hline \multicolumn{6}{|l|}{ ADM } \\
\hline Flexão & 0,058 & 53 & 0,023 & 0,099 & $0,011^{*}$ \\
\hline \multicolumn{6}{|l|}{ Força Muscular } \\
\hline $60^{\circ}$ & $-0,088$ & 22 & $-0,346$ & 0,229 & 0,610 \\
\hline $90^{\circ}$ & 0,122 & 38 & $-0,107$ & 0,569 & 0,308 \\
\hline $10^{\circ}$ & 0,180 & 34 & $-0,280$ & 0,910 & 0,541 \\
\hline \multicolumn{6}{|l|}{ WOMAC } \\
\hline Dor & 0,486 & 45 & 0,313 & 0,586 & $0,009 *$ \\
\hline Rigidez & 0,400 & 37 & $-0,075$ & 0,667 & 0,097 \\
\hline Função & 0,455 & 55 & 0,277 & 0,526 & $0,006^{*}$ \\
\hline Total & 0,482 & 54 & 0,261 & 0,620 & $0,008^{*}$ \\
\hline Funcionalidade & 0,159 & 33 & $-0,100$ & 0,469 & 0,236 \\
\hline
\end{tabular}

Est. Wilcoxon: valor crítico do teste de Wilcoxon; Lim. Inf. (IC): limite inferior do intervalo de confiança; Lim. Sup. (IC): limite superior do intervalo de confiança; AVD: atividades de vida diária ADM: amplitude de movimento; WOMAC: Western Ontario and McMaster Universities Osteoarthritis; *teste de Wilcoxon 
Tabela 4. Escores padronizados da dor, mobilidade, amplitude de movimento, força muscular, variáveis da Western Ontario and McMaster Universities Osteoarthritis e funcionalidade nos dois tempos de avaliação do Grupo Exercícios

\begin{tabular}{|c|c|c|c|c|c|}
\hline Variáveis & Mediana & Est. Wilcoxon & Lim. Inf. (IC95\%) & Lim. Sup. (IC95\%) & Valor $p$ \\
\hline \multicolumn{6}{|l|}{ Dor } \\
\hline AVD & 0,135 & 25 & $-0,165$ & 0,305 & 0,363 \\
\hline Repouso & 0,150 & 36 & $-0,183$ & 0,355 & 0,124 \\
\hline Mobilidade & 0,102 & 51 & 0,026 & 0,147 & $0,019^{*}$ \\
\hline \multicolumn{6}{|l|}{ ADM } \\
\hline Flexão & 0,044 & 34,5 & 0,010 & 0,110 & $0,025^{*}$ \\
\hline \multicolumn{6}{|l|}{ Força Muscular } \\
\hline $60^{\circ}$ & 0,108 & 34 & $-0,041$ & 0,493 & 0,193 \\
\hline $90^{\circ}$ & 0,025 & 28 & $-0,191$ & 0,256 & 1,000 \\
\hline $10^{\circ}$ & 0,049 & 31 & $-0,134$ & 0,301 & 0,760 \\
\hline \multicolumn{6}{|l|}{ WOMAC } \\
\hline Dor & 0,062 & 25 & $-0,038$ & 0,200 & 0,076 \\
\hline Rigidez & 0,256 & 32 & 0,000 & 0,525 & 0,059 \\
\hline Função & 0,073 & 35 & $-0,149$ & 0,280 & 0,476 \\
\hline Total & 0,055 & 36 & $-0,145$ & 0,297 & 0,415 \\
\hline Funcionalidade & $-0,029$ & 25 & $-0,249$ & 0,146 & 0,838 \\
\hline
\end{tabular}

Est. Wilcoxon: valor crítico do teste de Wilcoxon; Lim. Inf. (IC): limite inferior do intervalo de confiança; Lim. Sup. (IC): limite superior do intervalo de confiança; AVD: atividades de vida diária: ADM: amplitude de movimento; WOMAC: Western Ontario and MCMaster Universities Osteoarthritis; *teste de Wilcoxon

Tabela 5. Comparação entre os Grupos Ultrassom Contínuo, Ultrassom Pulsado e Exercícios para os escores padronizados das variáveis dor, mobilidade, amplitude de movimento, força muscular, variáveis da Western Ontario and McMaster Universities Osteoarthritis e funcionalidade nas avaliações 1 e 2

\begin{tabular}{|c|c|c|c|c|c|}
\hline Variáveis & USC $(n=10)$ & USP $(n=10)$ & $\operatorname{EXE}(n=10)$ & $H\left(\chi^{2}\right)$ & Valor $p$ \\
\hline \multicolumn{6}{|l|}{ Dor } \\
\hline AVD & $0,359^{a . b}$ & $0,514^{b}$ & $0,169^{a}$ & 7,13 & $0,03^{*}$ \\
\hline Repouso & $0,062^{\mathrm{a}}$ & $0,168^{a}$ & $0,180^{a}$ & 0,85 & 0,65 \\
\hline Mobilidade & $0,154^{a}$ & $0,131^{\mathrm{a}}$ & $0,117^{a}$ & 0,70 & 0,70 \\
\hline \multicolumn{6}{|l|}{ ADM } \\
\hline Flexão & $-0,02 \mathrm{O}^{\mathrm{a} . \mathrm{b}}$ & $-0,005^{a}$ & $0,098^{b}$ & 8,10 & $0,02^{*}$ \\
\hline \multicolumn{6}{|l|}{ Força Muscular } \\
\hline $60^{\circ}$ & $-0,029^{a}$ & $-0,150^{a}$ & $0,106^{a}$ & 2,68 & 0,26 \\
\hline $90^{\circ}$ & $0,106^{a}$ & $0,106^{a}$ & $-0,046^{a}$ & 0,95 & 0,62 \\
\hline $10^{\circ}$ & $-0,276^{a}$ & $0,011^{a}$ & $-0,065^{a}$ & 1,77 & 0,41 \\
\hline \multicolumn{6}{|l|}{ WOMAC } \\
\hline Dor & $0,402^{b}$ & $0,481^{b}$ & $0,040^{a}$ & 10,28 & $0,01^{*}$ \\
\hline Rigidez & $0,042^{\mathrm{a}}$ & $0,450^{a}$ & $0,256^{a}$ & 3,03 & 0,22 \\
\hline Função & $0,400^{a, b}$ & $0,486^{b}$ & $0,090^{\mathrm{a}}$ & 7,34 & $0,03^{*}$ \\
\hline Total & $0,372^{\mathrm{a}, \mathrm{b}}$ & $0,483^{b}$ & $0,055^{\mathrm{a}}$ & 9,20 & $0,01^{*}$ \\
\hline Funcionalidade & $0,036^{a}$ & $0,058^{a}$ & $0,038^{a}$ & 1,14 & 0,57 \\
\hline
\end{tabular}

H: valor crítico do teste de Kruskall-Walis; ab.indicam diferença estatística entre os tratamentos, pelo teste de múltipla comparação de Dunn; Valor Crítico ( $\chi^{2} ; 2 \mathrm{gl} ; 0,05$ )=5,99; AVD: atividades de vida diária; ADM: amplitude de movimento; WOMAC: Western Ontario and McMaster Universities Osteoarthritis; *teste de Kruskall-Walis

repouso (Dor rep), WOMAC função e WOMAC total, quando comparados ao USP. O Grupo EXE foi melhor que ambos os grupos tratados com US na melhora da WOMAC dor.

Nas tabelas, para as variáveis $\mathrm{ADM}$ e FM, foram apresentados os valores dos joelhos direitos. Os resultados deste não foram significativamente diferentes $(p>0,05)$ do que os dos joelhos esquerdos.

\section{DISCUSSÃO}

A OA tem grande incidência em idosos e maior prevalência no sexo feminino ${ }^{18}$. Pudemos observar, em nosso estudo, uma maior participação de mulheres acima de 60 anos. Segundo Ding ${ }^{19}$, as mulheres realizam mais consultas médicas e entre as principais queixas encontram-se as doenças crônico-degenerativas, como a OA. 
Outro fator de prevalência bastante relatado em outros estudos ${ }^{20}$, e também comprovado no nosso, é o elevado IMC nos pacientes com OA de joelho, sendo um dos principais responsáveis pela dor.

Neste estudo foi observado um alívio da dor, melhora da função e da QV em ambos os grupos tratados com o US. Acredita-se que a analgesia encontrada em ambos os grupos, tenha ocorrido devido ao controle da inflamação periarticular causada pelo US aplicado em pontos específicos preconizados pela World Association of Laser Therapy ${ }^{21}$ sobre a cápsula articular.

Em relação ao exercício físico, existem evidências científicas que apontam para os efeitos favoráveis na dor, na mobilidade e na função de pacientes com OA de joelho ${ }^{8-10}$. Neste estudo foi verificada, no Grupo EXE, uma melhora na mobilidade dos pacientes. Acredita-se que a analgesia encontrada em ambos os grupos tratados com US associado a exercícios tenha sido provocada pelo US. Essa analgesia pode ter favorecido uma melhor execução dos exercícios por parte dos pacientes, acentuado os ganhos funcionais.

O Grupo USP também apresentou melhora na dor. Segundo $\mathrm{Ng}$ et al. ${ }^{22}$, a diminuição da rigidez e melhora na ADM provocada pelo o US pulsado pode ser em decorrência dos seus efeitos em modular a produção de fibroblastos, a síntese de colágeno, quebrar a adesão tecidual e acelerar a cicatrização.

Pacientes com OA de joelho apresentam uma redução de 50-60\% da força de quadríceps, causada por atrofia por desuso e inibição artrogênica ${ }^{23}$. Estudo ${ }^{24}$ tem proposto que a disfunção sensório-motora do quadríceps pode ser importante na patogênese e na progressão da OA, como um determinante da inabilidade. Nossos resultados mostraram que houve uma melhora significativa da força do músculo quadríceps no grupo USP.

$\mathrm{Na}$ comparação entre os grupos contatou-se que os grupos USC e EXE foram mais eficazes na melhora da dor, na ADM, função e QV do que o grupo USP. A redução da dor de tecidos moles causada pelo US contínuo se deve a um aumento da temperatura e do fluxo sanguíneo nos músculos com espasmos, causando o relaxamento desses.

Ozgönenel et al. ${ }^{25}$ encontraram uma melhora na QV e mobilidade de pacientes com OA de joelho que receberam aplicação de US contínuo quando comparado ao grupo placebo. Já Tascioglu et al. ${ }^{26}$ concluíram que o US pulsado é mais efetivo no alívio da dor e na $\mathrm{QV}$ de pacientes com OA de joelho.

Cetin et al. ${ }^{27}$, assim como no presente estudo, concluíram que a aplicação do US antes do exercício ocasiona um aumento do desempenho na realização do exercício, diminuição da dor e melhora da função. Huang et al. ${ }^{5}$ concluíram que especialmente o US pulsado pode aumentar os efeitos terapêuticos dos exercícios isocinéticos no alívio da dor e melhora da função de pacientes com OA de joelho. Diferente desses, Falconer et al. ${ }^{28}$ avaliaram a eficácia do US em pacientes com OA de joelho com contraturas crônicas. Os autores sugeriram que apesar de o US não poder contribuir com o tratamento de pacientes com rigidez crônica de joelhos com OA, os benefícios do programa de exercícios, provavelmente, influenciou a melhoria global dos pacientes.

\section{CONCLUSÃO}

Este estudo mostra que a associação do USC a exercícios foi mais efetiva na melhora da dor, da mobilidade articular, funcionalidade e $\mathrm{QV}$ em pacientes com OA de joelho, quando comparada à associação do USP a exercícios ou à aplicação isolada de exercícios.

\section{REFERÊNCIAS}

1. Badley E, Tennant A. Impact of disablement due to rheumatic disorders in a British population: estimates of severity and prevalence from the Calderdale Rheumatic Disablement Survey. Ann Rheum Dis. 1993;52(1):6-13.

2. Jordan JM, Renner JB, Luta G, Dragomir A, Fryer JG, Helmic C, et al. Hip osteoarthritis is not rare in African-Americans and is different than in Caucasians. Arthritis Rheum. 1997:40(suppl):5236.

3. [Prevalência de osteoartrite no Brasil [cited 2011 Jan]. Available from: http://www.reumatologia.com.br

4. Ricci NA, Coimbra IB. Exercício físico como tratamento na osteoartrite de quadril: uma revisão de ensaios clínicos aleatórios controlados. Rev Bras Reumatol. 2006;46(4):273-80.

5. Huang MH, Lin YS, Lee CL, Yang RC. Use of ultrasound to increase effectiveness of isokinetic exercise for knee osteoarthritis. Arch Phys Med Rehabil. 2005;86(8):1545-51.

6. Ettinger WH, Afable RF. Physical disability from knee osteoarthritis: the role of exercise as an intervention. Med Sci Sport Exerc. 1994:26(12):1435-40.

7. Pelletier JP, Martel-Pelletier J, Abramson SB. Osteoarthritis, an inflammatory disease: potential implication for the selection of new therapeutic targets. Arthritis Rheum. 2001;44(6):1237-47.

8. Otawa panel evidence-based clinical practice guidelines for therapeutic exercises and manual therapy in the management of osteoarthritis. Phys Ther. 2005:85(9):907-71.

9. Jordan K, Arden N, Doherty M, Bannwarth B, Bijlsma JW, Dieppe P, et al. EULAR recommendations 2003: an evidence based approach to the management of knee osteoarthritis. Report of a Task Force 
on the Standing Committee for International Clinical Studies Including Therapeutic Trials (ESCISIT). Ann Rheum Dis. 2003;62(12): 1145-55.

10. Recommendations for the medical management of osteoarthritis of the hip and knee: 2000 update. American College of Rheumatology Subcommittee on Osteoarthritis Guidelines. Arthritis Rheum. 2000;43(9):1905-15

11. Kellgren JH, Lawrence JS. Radiological assessment of osteo-arthrosis. Ann Rheum Dis. 1957;16(4):494-501.

12. Bellamy N, Buchnan WW, Goldsmith CH, Campbell J, Stitt LW. Validation study of WOMAC: a health status instrument for measuring clinically important patient relevant outcomes to antirheumatic drug therapy in patients with osteoarthritis of the hip or knee. J Rheumatol. 1988:15(12):1833-40.

13. Lequesne MG. The algofunctional indices for hip and knee osteoarthritis. J Rheumatology. 1997;24(4):764-802.

14. Revill SI, Robinson JO, Rosen M, Hogg IJ. The reliability of a linear analogue for evaluating pain. Anesthesia. 1976;31(9):1191-8.

15. Marques PA. Manual de goniometria. 2a ed. Barueri: Editora Manole; 2003.

16. Bandinelli S, Benvenuti E, Del Lungo I, Baccini M, Benvenuti F, Di lorio A, et al. Measuring muscular strength of the lower limbs by handheld dynamometer: a standard protocol. Aging (Milano). 1999;11(5): 287-93.

17. Piva SR, Fitzgerald GK, Irrgang IJ, Bouzubar F, Starz TW. Get up and go test in patients with knee osteoarthritis. Arch Phys Med Reabil. 2004;85(2):284-9.

18. Kitchen SS, Partridge CJ. A review of therapeutic ultrasound, part 1: background and physiological effects. Physiotherapy.1990;76:593-5.

19. Ding C. Sex differences in knee cartilage volume in adults: role of body and bone size, age and physical activity. Rheumatology (Oxford). 2003;42(11):1317-23.
20. Toivanen AT, Heliövaara M, Impivaara O, Arokoski JP, Knekt P, Lauren $\mathrm{H}$, et al. Obesity, physically demanding work and traumatic knee injury are major risk factors for knee osteoarthritis - a populationbased study with a follow-up of 22 years. Rheumatolology (Oxford). 2010:49(2):308-14.

21. World Association of Laser Therapy (WALT). Consensus agreement on the design and conduct of clinical studies with low-level laser therapy and light therapy for musculoskeletal pain and disorders. Photomed Laser Surg. 2006;24(6):761-2.

22. Ng CO, Ng GY, See EK, Leung MC. Therapeutic ultrasound improves strength of Achilles tendon repair in rats. Ultrasound Med Biol. 2003;29(10):1501-6

23. Hassan B, Mockett S, Doherty M. Static postural sway, proprioception, and maximal voluntary quadriceps contraction in patient with knee osteoarthritis and normal control subjects. Ann Rheum Dis. 2001;60(6):612-8.

24. Slemenda C, Brandt KD, Heilman DK, Mazzuca S, Braunstein EM, Katz BP, et al. Quadriceps weakness and osteoarthritis of the knee. Ann Inter Med. 1997:127(2):97-104.

25. Ozgönenel L, Aytekin E, Durmuşoglu G. A double-blind trial of clinical effects of therapeutic ultrasound in knee osteoarthritis. Ultrasound Med Biol. 2009;35(1):44-9.

26. Tascioglu F, Kuzgun S, Armagan O, Ogutler G. Short-term effectiveness of ultrasound therapy in knee osteoarthritis. J Int Med Res. 2010;38(4):1233-42.

27. Cetin N, Aytar A, Atalay A, Akman MN. Comparing hot pack, shortwave diathermy, ultrasound, and TENS on isokinetic strength, pain, and functional status of women with osteoarthritic knees: a single-blind, randomized, controlledtrial. Am J Phys Med Rehabil. 2008;87(6):443-5.

28. Falconer J, Hayes KW, Chang RW. Therapeutic ultrasound in the treatment of musculoskeletal conditions. Arthritis Care Res. 1990;3(2):85-91. 